\title{
Discriminate Vertical Handoff Time Using GRA and TOPSIS Algorithms in Heterogeneous Networks by Effect of Metrics
}

\author{
"M Naresh ${ }^{1}$, Dr D Venkat Reddy ${ }^{2}$, Dr K Ramalinga Reddy ${ }^{3}$ \\ ${ }^{1}$ Assistant Professor, ECE Dept., Matrusri Engineering College, Hyderabad \\ ${ }^{2}$ Professor, ECE Dept., Mahatma Gandhi Institute of Technology, Hyderabad \\ ${ }^{3}$ Professor \& HOD, ETM Dept., G Narayanamma Institute of Technology and Science for Women, Hyderabad \\ Corresponding Author: M Naresh
}

\begin{abstract}
Coherent persistence is the main objective and has been a vital challenge in fourth generation wireless networks (FGWNs). "HANDOVER" is the best possible solution to achieve the coherent connectivity, which will be used for service persistence, when a mobile terminal (MT) is in overlapping area. To support coherent persistence in heterogeneous networks, the traditional Vertical Handover Management (VHM) approaches are not good enough. Hence, it is very important to deal with those challenges like selection of network and triggering of incompatible handover. In Heterogeneous wireless networks main test is constant association among the different networks like Wi-Fi, WI-Max, WLAN and WPAN etc. In this proposal, various Vertical handover decision techniques and schemes have been compared such as, Gray Relational Analysis (GRA) and Technique for Order Preference by Similarity to Ideal Solution (TOPSIS). These approaches helps for the consistent connectivity form the mobile terminal by choosing the best network among the Visitor networks (VTs) those are available. Our proposal work mainly focused on that at the time of handover, the handover decision phase as well as to mitigate the processing delay. We also compared both the GRA and TOPSIS methods with the Quality of Service (QoS) parameters of the mobile terminal (MT) to acquire the connectivity with the best network.
\end{abstract}

Keywords: Handover mechanism, vertical handover management (VHM), vertical handoff decision (VHD), gray relational analysis (GRA) and TOPSIS

Date of Submission: 13-07-2017

Date of acceptance: $15-07-2017$

\section{INTRODUCTION}

A Wireless mobile is not just used for communication, also used for data transfer, video conferencing, net-surfing etc,. For all these utility, high bandwidth and rapid transfer of data is required. In case of versatility of a wireless gadget, signal gathering will be a test in dense areas. In this crossroads it is prescribed to afford different networks. Each network will have a scope of coverage. At the point when a wireless hub moves past its cell-impediment, it should change over to another base station which belongs to different network. Since its old network never again supports it. This is named as heterogeneous-handoff. In case of Horizontal handoff, which involves the same network, Received signal strength measure is the main significant thing to consider. For the heterogeneity, we have to investigate different parameters such as bandwidth, delay, Signal to Noise ratio etc. We should also consider the User preferences such as wide coverage, low cost, security etc. At the point when a user using a low-cost network is given over to another high-cost network, at that point it will be an issue. So we need to break down different types of is-sues while considering heterogeneous network. In fourth generation wireless networks, service continuity is a main goal i.e., when a MT or mobile hub (MN) moving in an overlapping area, continuous service must be required so that the "HANDOVER" technique can be applicable. The handover technique is mainly used to redirect the mobile user's service network from one base station (BS) to another BS as current network to another network or one access point (AP) to another AP with same technology or among different technologies to reduce the processing delay in the overlapping area. We have two types of Handover technique they are horizontal handover and vertical handover. Horizontal handover is performed by the homogenous wireless network and if there are two base stations's (BS) using the same access technology, in a current system that technology is called horizontal handover. For surrounding BSs to trigger and to perform the handover decision, horizontal handover mechanism uses signal strength measurements. Various network interfaces will be equipped by the mobile station (MS) or Base station (BS) to achieve different wireless networks in 
heterogeneous wireless networks. At the point when an emerging blend of over lapping heterogeneous wireless networks conveyed, vertical handover is used among the networks using different access technologies. The main focus and scope of our proposal is in handover decision phase, which will chose the best network from the networks those are available as mentioned by the decision phase. In this proposal, the best decision makers and the target visitor network (TVN) selector from the various visitors' networks are GRA and TOPSIS. And TOPSIS method is utilized for proposed decision making in a distributed manner and also compared with the GRA method. There are several parameters took by the MT such as bandwidth, delay, throughput, Delivery ratio and energy as the decision parameters for handover.

\section{RELATED WORK}

Faouzizarai in [3] formulated the architecture for handoff decision making which considers the resource use and the user inclination for the network. Integrated frame-work through multilayer has been proposed by the author in [4], which genius vides a holistic handoff solution to the impromptu networks. Then after a scheme proposed in [5] requires a pre network registration and authentication before handoff. It also used parcel buffering technique for keeping the bundle loss. Lahby Mohammed et al [6] gave a different approach by combining the ANP and TOPSIS methods. Their approach also given by considering and disposing of the differentiated weights of criterions and history attributes for handoff. Nirmala Shenoy [7] presented a framework for seamless meandering which overlays network comprising of Inter-System Interface Control Units (IICU) to support internetwork communication and control for Location Management. It optimizes the call delays, traffic and different QOS para-meters. Y.Wu et al [8] experimented a congestion mindful vertical handoff which reduced the bundle loss and delay. The algorithm names as vertical handoff decision (VHD) has been discussed in [9] for heterogeneous wireless networks. The author in [9] formulated that the issue as a Markov decision process. The same has formulated as fuzzy multiple attribute decision making (MADM) in [10]. The authors in [12] implemented a new scheme named as vertical handoff decision scheme that have been motivated by mitigation of overload and processing delay at the mobile terminal. The scheme proposed in [12] will reduce the consumption of power as well as processing delay. In [13], the author is mainly used to decrease the processing delay and to settle on a trust handoff decision in a heterogeneous wireless condition using Trusted-Distributed-VHD. In [14] a novel distributed vertical handoff decision scheme using the SAW method with a distributed manner to maintain a strategic distance from the drawbacks. In [15], it provides the four steps integrated strategy for MADM based network selection to solve the issue. All these proposal works are mainly focused on the handoff decision and ascertain the handoff decision criteria on the mobile terminal side and the discussed scheme are used to reduce the processing delay by the estimation process using MADM in a distributed manner [16].

\section{CONVENTIONAL SCHEMES}

\section{A. Gray Relational Analysis}

The main method of GRA [16] is firstly translating the performance of all alternatives into a similarity sequence, which is known as gray relational generating and a reference sequence will be characterized according these sequences at which, the gray relational coefficient has been calculated between the reference sequence and all equivalence sequences. Finally, the gray relation review will be calculated between the each similarity sequences and reference sequence based on these obtained gray relational coefficients. On the off chance that a similarity sequence translated from an option has the highest gray relational review between the reference sequence and itself, that option will be the best decision. The procedures of gray relational analysis are shown in Fig 1. Network instatement process starts, here mobile node request for handoff. In this network, any network is available means broadcasting happen for handoff process based on network parameters. Ascertain the weight factor for the available networks at that point select the network which has high weight factor. So send request to that network. We can know the handoff process finished. When handoff process finished then mobile node send the data to particular network. The handoff process happen based on methodology and parameters like QoS. At the point when mobile node handoff request time network is not available means end the process it resembles mobile node continuously tries for new network.

\section{1) Gray relational generating}

At the point when the units in which performance is measured are different for different attributes, the impact of some attributes might be ignored. This may also happen if some performance attributes have a substantial range. In addition, incorrect results will be caused in the analysis if there are different attributes directions and goals [16]. Hence, it is necessary to process all the every alternative performance value into a sequence of comparability in analog to normalization process, which is called as gray relational generating in GRA and this will be suitable for undiscovered problems with poor information also provides the correlation 
between the best ideal network and candidate network [17].

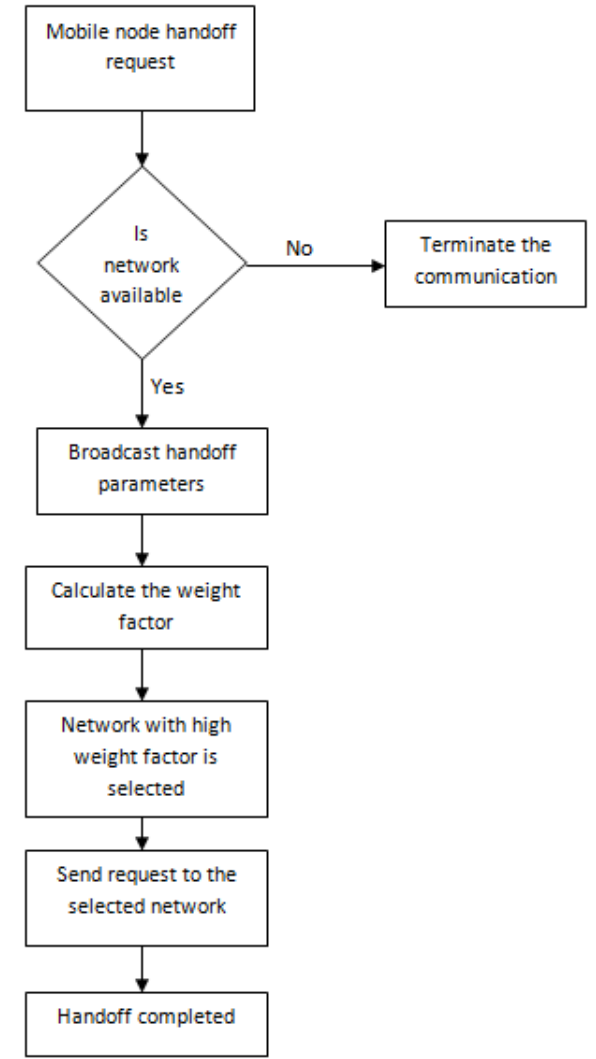

Fig.1 Flowchart of grey rational analysis

Here, gray relational coefficient (GRC) is calculated, before that normalization of data and characterizing ideal sequence. Normalization of data is done for three situations bigger the better, smaller the better, nominal the best.

$$
\begin{aligned}
& \mathrm{N}_{\mathrm{ij}}=\mathrm{P}_{\mathrm{ij}}-\min \left(\mathrm{P}_{\mathrm{ij}}\right) /\left(\min \left(\mathrm{P}_{\mathrm{ij}}\right)-\max \left(\mathrm{P}_{\mathrm{ij}}\right)\right) \\
& \mathrm{N}_{\mathrm{ij}}=\max \left(\mathrm{P}_{\mathrm{ij}}\right)-\mathrm{P}_{\mathrm{ij}} /\left(\max \left(\mathrm{P}_{\mathrm{ij}}\right)-\min \left(\mathrm{P}_{\mathrm{ij}}\right)\right)
\end{aligned}
$$$$
\mathrm{N}_{\mathrm{ij}}=1-\left|\mathrm{P}_{\mathrm{ij}}-\mathrm{m}_{\mathrm{j}}\right| / \max \left\{\max -\mathrm{m}_{\mathrm{j}}, \mathrm{m}_{\mathrm{j}}-\min \left(\mathrm{P}_{\mathrm{ij}}\right)\right\}
$$
$\mathrm{m}_{\mathrm{j}}$ is the largest value in the nominal the best situations.

$$
\begin{gathered}
\Delta_{\mathrm{i}}=\left|\mathrm{P}_{0 \mathrm{j}}-\mathrm{N}_{\mathrm{ij}}\right| \quad, \Delta_{\max }=\max _{i \in \boldsymbol{M}, j \in N} \Delta_{i} \\
\Delta_{\min }=\min _{i \in M, j \in N} \Delta_{i} \\
\mathrm{GRC}_{\mathrm{i}}=1 / \mathrm{m} \sum_{\mathrm{j}=1}^{\mathrm{m}}\left(\Delta_{\max }+\Delta_{\min /\left(\Delta_{i}+\Delta_{\max }\right)}\right) \\
\text { Score }_{\mathrm{GRA}}=\arg \max _{i \in M} \mathrm{GRC}_{\mathrm{i}}
\end{gathered}
$$

\section{PROPOSED FRAMEWORK}

Our proposed method considered and analyzed the TOPSIS scheme, in which there are three types of attributes. Those follows:

- Soft benefit attributes/ criteria

- Quantitative benefit attributes

- Cost attributes
The main concept of this approach is that it should have shortest distance for chosen alternative from the positive ideal solution and the same alterative has the farthest distance from the negative ideal solution.

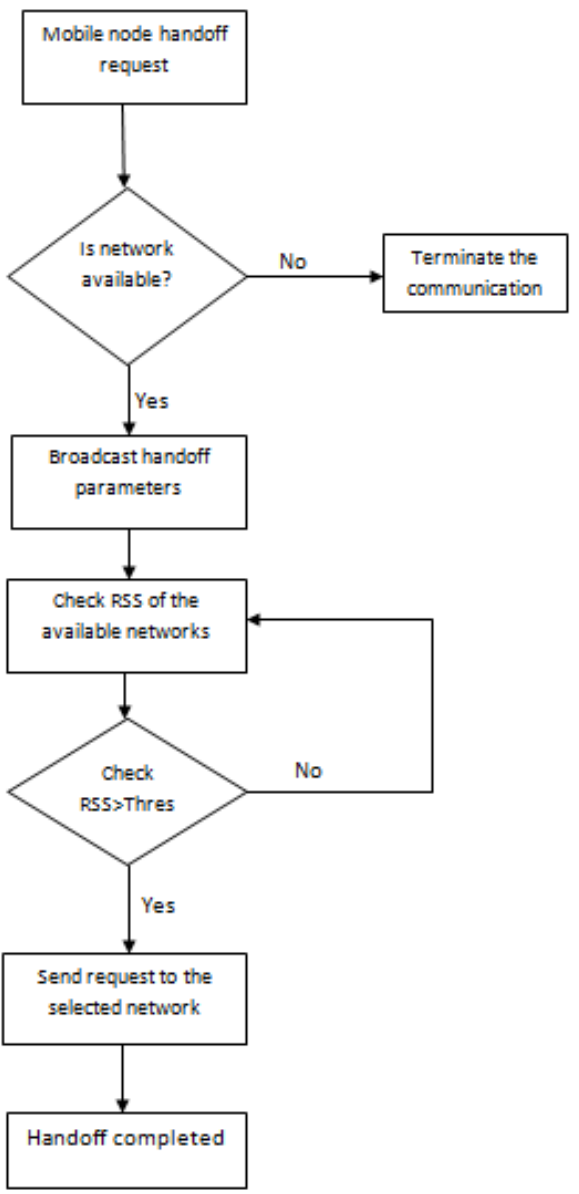

Fig.2 Proposed flowchart

TOPSIS [4] is a method that selects a network by satisfying the criteria given after performing the below listed sequential steps. The network with maximum value from the rank order is the one that is close to the positive ideal solution and far away from the negative solution. The criteria for selecting the network are maximum bandwidth, handoff signaling delay, Received signal strength and minimum cost. To find the rank of network we this technique follows the step as below:

1. To construct normalized decision matrix consisting of various attributes such as Bandwidth, delay, Jitter, Packet loss etc $\mathrm{N}_{\mathrm{ij}}=\mathrm{P}_{\mathrm{ij}} / \sqrt{ } \sum_{\mathrm{m}}^{\mathrm{i}=1} \mathrm{P}_{\mathrm{ij}}^{2}$

2. To compute weighted normalized matrix by $\mathrm{V}_{\mathrm{ij}}=\mathrm{N}_{\mathrm{ij}} * \mathrm{~W}_{\mathrm{j}}$

3. To find positive and negative ideal solution $\mathrm{A}^{+}=\left\{\left(\max V_{\mathrm{ij}} / \mathrm{j} \epsilon \mathrm{J}\right),\left(\min V_{\mathrm{ij}} / \mathrm{j} \epsilon \mathrm{J}^{\prime}\right)\right\}, \quad \mathrm{A}^{-}=$

$\left\{\left(\operatorname{minV}_{\mathrm{ij}} / \mathrm{j} \Theta \mathrm{J}\right),\left(\operatorname{maxV}_{\mathrm{ij}} / \mathrm{j} \mathrm{J}^{\prime}\right)\right\}$ where $\mathrm{J}$ is a set of benefit parameters and J' is a set of cost parameters

4. Calculate the separation distance between available networks and positive and negative ideal solutions. 


$$
\mathrm{S}^{+}=\sqrt{\sum_{\mathrm{j} \in \mathrm{N}}\left(\mathrm{V}_{\mathrm{ij}}-\mathrm{V}_{\mathrm{j}}^{+}\right)^{2}}, \mathrm{~S}^{-}=\sqrt{\sum_{\mathrm{j} \in \mathrm{N}}\left(\mathrm{V}_{\mathrm{ij}}-\mathrm{V}_{\mathrm{j}}^{-}\right)^{2}}
$$

5. Compute the closeness to ideal solution $\mathrm{C}_{\mathrm{i}}=$ $\mathrm{S}^{-} /\left(\mathrm{S}^{+}+\mathrm{S}^{-}\right)$, Score $_{\mathrm{TOPSIS}}=\arg \max _{i \in M} \mathrm{C}_{\mathrm{i}}$

Network initialization process starts, here mobile node request for handoff. In this network, any network is available means broadcasting happen for handoff process based on network parameters. Ascertain the weight factor for the available networks with got signal strength calculates then select the network which has high weight factor with state of RSS is greater than threshold esteem. So send request to that network. Here RSS is high efficient and effective of network. It is an effective parameter and measure the high performance. We can know the handoff process finished. When handoff process finished then mobile node send the data to particular network. The handoff process happen based on methodology and parameters like Qos. At the point when mobile node handoff request time network is not available means end the communication process it resembles mobile node continuously tries for new network. In this RSS condition is fails on the other hand check the RSS of remaining network what are available.

\section{RESULTS AND DISCUSSION}

The simulation process starts with the network process with vertical handoff. Here the mobile node indicates how to request the handoff in network with support of methodology. The process of simulation begins through some parameters and library files with object files. The simulation carried out in a network simulator (NS-2). Here NOAH and DSDV routing protocols used. NOAH supports for GRA and DSDV supports for TOPSIS

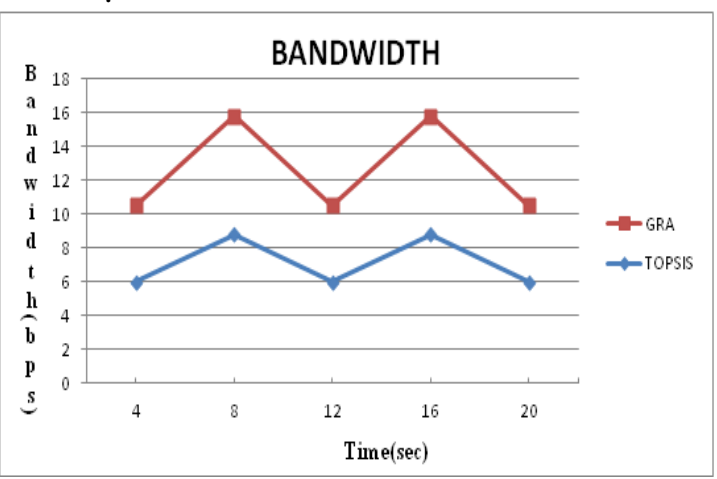

Fig. 3 Performance comparison of bandwidth

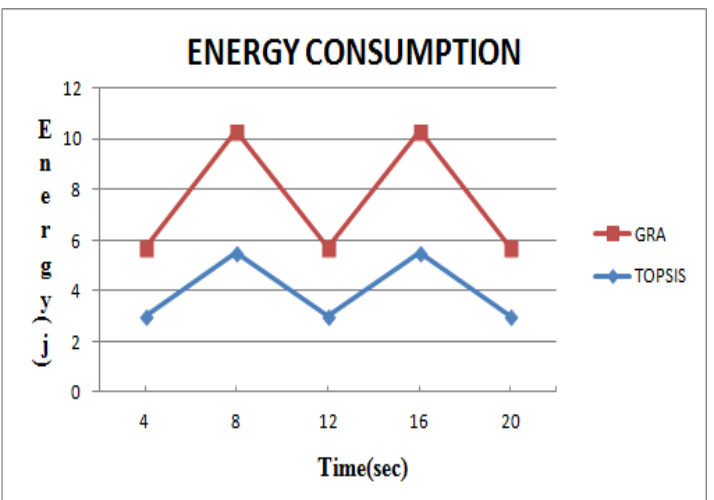

Fig. 4 Comparison if energy consumption

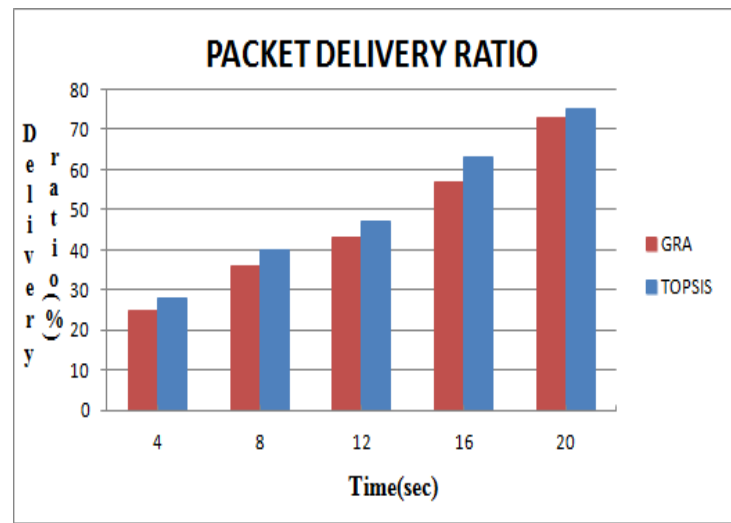

Fig. 5 Packet delivery ratio analysis

To evaluate the proposed approach compare with existing approach, five performance metrics are considered in the experiments: 1) Bandwidth: data measured bit per sec. 2) Energy consumption: Individual energy levels of node calculates based on time interval. 3) Packet delivery ratio: how much data delivery from initialization to ending of the process. 4) Delay: The nodes travelling some distance based on time functionality. And 5) Throughput: total data transmission based on simulation time by measuring the bit per sec process. Figure (3), (4), (5), (6) and (7) represents the bandwidth, energy consumption, packet delivery ratio, delay and throughput parameters with respect to time.

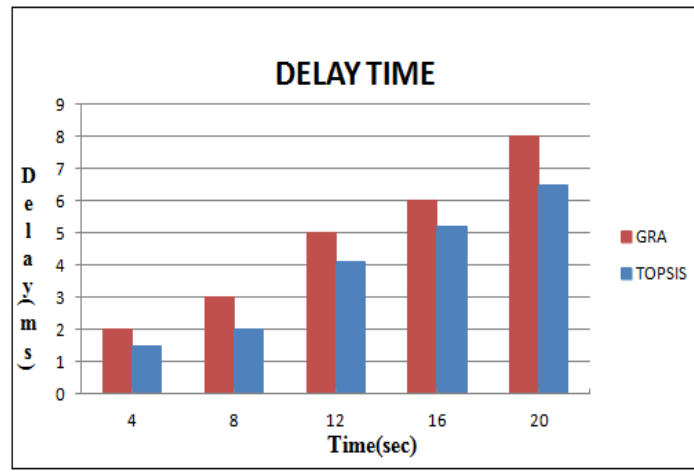

Fig. 6 Performance comparison of delay time 


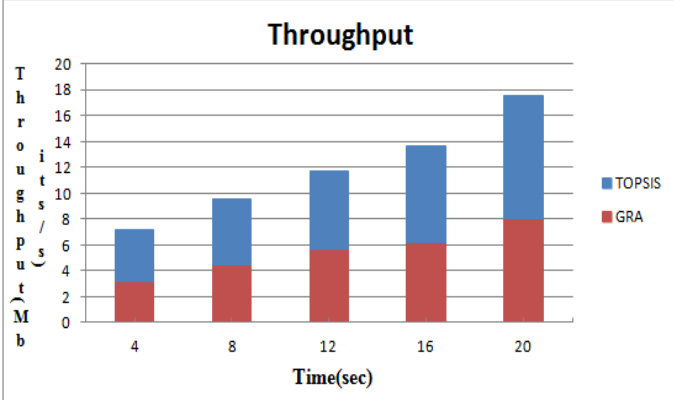

Fig. 7 Comparison of throughput values with the various time intervals

These graphs measure the system process and compared with the existing algorithm and showed that our proposed system has more effective performance than GRA.

Table I Simulation Parameters

\begin{tabular}{ll}
\hline PARAMETER & VALUE \\
\hline Application traffic & CBR \\
Transmission rate & 10 packets/sec \\
Radio range & $250 \mathrm{~m}$ \\
Packet size & 1000 bytes \\
Channel data rate & $15 \mathrm{Mbps}$ \\
Maximum speed & $50 \mathrm{~m} / \mathrm{s}$ \\
Simulation time & $20 \mathrm{secs}$ \\
Number of nodes & 8 \\
Area & $1000 x 1000$ \\
Network & $802 \_11,802 \_16$ \\
Method & GRA,TOPSIS \\
Routing protocol & NOAH,DSDV \\
\hline
\end{tabular}

\section{CONCLUSION}

In our proposal, VHD schems have been compared in the heterogeneous wireless networks. The observation of schemes has been done to mitigate the processing delay and a trusted handover decision. In this paper we proposed decision makers GRA and TOPSIS to select the best network from the visitor network for the Vertical decision schemes. The best decision maker is analyzed by the relative standard deviation and the best one is TOPSIS. Our main goal is in the decision phase of the handover phases to take decision to which $\mathrm{VN}$ the mobile terminal to associate with decrease the processing delay by different decision algorithms.

\section{REFERENCES}

[1] Anany Levitin, "Introduction to the Design and Analysis of Algorithms", 3rd Edition Pearson Publications, pp: 41-50, 2012.

[2] Ermatita, "MADM Methods in Solving Group Decision Support System on Gene Mutations Detection Simulation”, International Conference on Distributed
Framework and Applications (DFmA), pp.16, Aug 2010.

[3] Faouzi zarai "Seamless Mobility in Heterogeneous Wireless Networks", International Journal of Next Generation Network (IJNGN), Volume 2, No 4, Dec 2010.

[4] Hui zeng, "A Multi Layer approach for Seamless Soft Handoff for Mobile Ad Hoc Networks", IEEE Globecom Workshops, Dec 2010

[5] Hyun-ho choi, "Seamless Handoff Scheme based on Pre-registration and Preauthentication of UMTS-WLAN interworking", Wireless Personal Communications, Springer, Vol. 41, pp. 345-364 2007.

[6] Lahby Mohammed, “An Intelligent Network Selection Strategy Based on MADM Methods in Heterogeneous Net-works", International Journal of Wireless and Mobile Networks (IJWMN), Volume 4, No 1, Feb 2012.

[7] Nirmala shenoy, "A Framework for Seamless Roaming Across Heterogeneous Next Generation Wireless Networks", Wireless Networks, Volume 11, pp. 757774, Springer science 2005.

[8] Y.Wu, "Congestion-aware proactive vertical handoff algorithm in heterogeneous wireless networks", IET communications, Vol. 3, Issue 7, pp. 1103-1114, 2009

[9] E.Steven-Navarro, V.W.S. Wong and Yuxia Lin, "A Vertical Handoff Decision Algorithm For Heterogeneous Wireless Networks", Wireless Communications and Networking Conference, IEEE, Kowloon pp. 3199- 3204, 2007.

[10] Wenhui Zhang, "Handover Decision Using Fuzzy MADM in Heterogeneous Networks", Wireless Communications and Networking Conference, IEEE, Vol.2, pp. 653-658, 2004.

[11] R.Tawil, G.Pujolle and O.Salazar, "A Vertical Handoff Decision Schemes In Heterogeneous Wireless Systems", Vehicular Technology Conference, VTC Spring IEEE, Singapore, pp. 2626-2630, 2008.

[12] R.Tawil, J.Demerjain, G.Pujolle And O.Salazar, "Processing-Delay Reduction During The Vertical Handoff Decision In Heterogeneous Wireless System", International Conference on Computer Systems and Applications, AICCSA IEEE/ACS , pp.381-385, 2008.

[13] R.Tawil, J.Demerjain and G.Pujolle, "A Trusted Handoff Decision Scheme for the 
Next Generation Wireless Networks", IJCSNS, Vol.8, pp.174-182, June 2008.

[14] R.Tawil, G.Pujolle and O.Salazar, "Vertical Handoff Decision Schemes dor The Next Generation Wireless Networks", Wireless Communications and Networking Conference, pp. 2789-2792, 2008.

[15] Lusheng Wang and David Binet, "MADMBased Network Selection in Heterogeneous Wireless Networks: A Simulation Study", 1st International Conference on Wireless Communication, Vehicular Technology, Information Theory and Aerospace \& Electronic Systems Technology, Wireless VITAE, pp. 559-564, 2009.

[16] Yiyo Kuo, "The use of grey relational analysis in solving multiple attribute decision-making problems", Elsevier Publications, Volume 55, Issue 1, pp. 80-93, August 2008

[17] Enrique Stevens-Navarro and Vincent W.S. Wong "Comparison between Vertical Handoff Decision Algorithms for Heterogeneous Wireless Networks", IEEE, pp.947-951, 2006

\footnotetext{
International Journal of Engineering Research and Applications (IJERA) is UGC approved Journal with Sl. No. 4525, Journal no. 47088. Indexed in Cross Ref, Index Copernicus (ICV 80.82), NASA, Ads, Researcher Id Thomson Reuters, DOAJ.

M Naresh. "Discriminate Vertical Handoff Time Using GRA and TOPSIS Algorithms in Heterogeneous Networks by Effect of Metrics." International Journal of Engineering Research and Applications (IJERA) 7.7 (2017): 49-54.
} 